\title{
Produktionsraten und metabolische Clearanceraten von Östrogenen und von Progesteron beim Menschen ${ }^{1)}$
}

\author{
Von LIESELOTTE NOCKe-FINCK und H. BREUER \\ Institut für Klinische Biochemie und Klinische Chemie der Universität Bonn
}

(Eingegangen am 6. Mai 1972)

Die Bestimmung der Produktionsraten von Ostrogenen und Progesteron kann mit Hilfe von 2 Verfahren crfolgen: Die „Urin“-Produktionsrate und die „Blut"-Produktionsrate. Die „Urin“-Produktionstate (PR Urin) wird nach der Formel

$$
P_{U \text { Urin }}=\frac{\text { injiziertes radioaktiv markiertes Steroid }}{\text { spezif. Aktivität des Metaboliten }} \times \frac{1}{t},
$$

die „Blut“-Produktionsrate (PR Blut) nach der Formel

$$
\mathrm{PR}_{\mathrm{Blut}}=\mathrm{MCR} \times \mathrm{C}
$$

berechnet, wobei MCR die metabolische Clearancerate und C die Konzentration des endogenen Steroids im Plasma bedeuten. Die Aussage$1 \mathrm{kraft}$ der PR Urin von Östrogenen ist mit gewissen Unsicherheiten belastet, da diese Steroide einem enterohepatischen Kreislauf unteriegen, ferner im Organismus eine Interkonversion von Ostron und Ostradiol-17 $\beta$ erfolgt und schließlich das Ausmaß der Umwandlung von Androgenen in Ostrogene von mehreren Faktoren abhängig ist; außerdem spielen Nieren- und Leberfunktion eine wichtige Rolle. Die PR Urin für Ostradiol-17 $\beta$ und Ostron ist bei nichtschwangeren Frauen während der Follikelphase niedriger als während der Lutealphase; bei Männern liegen die Werte deutlich niedriger als bei Frauen in der Reproduktionsphase. Am Ende der Schwangerschaft beträgt die PR Urin für Östradiol-17 $\beta$ und Ostron zusammen bis zu $30 \mathrm{mg} / \mathrm{Tag}$. Die $\mathrm{PR}_{\mathrm{Blut}}$ ist wegen der relativ schwierigen Bestimmung der endogenen Östrogene nur in wenigen Fällen ermittelt worden.

Die bisherigen Untersuchungen zur Bestimmung der metabolischen Clearance-Rate sind unter Verwendung der kontinuierlichen Infusionstechnik durchgeführt worden. Die metabolische Clearance-Rate von Östron ist bei Männern und Frauen etwa gleich groß, während sie für Östradiol-17 $\beta$ bei Männern signifikant höher ist als bei Frauen. Die Größe der metabolischen Clearance-Rate ist abhängig vom hormonellen Status.

Die Berechnung der PR $R_{\text {rin }}$ von Progesteron basiert auf der Ausscheidung von 5 $\beta$-Pregnan-3 $\alpha, 20 \alpha$-diol, dem Hauptmetaboliten vom Progesteron. Die Bestimmung der PR Urin von Progesteron hat nur während der Schwangerschaft als „Screening“-Test einen Aussage-

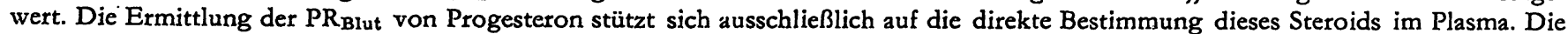

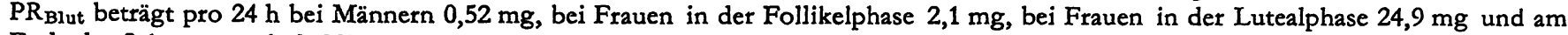
Ende der Schwangerschaft $293 \mathrm{mg}$.

Die metabolischen Clearance-Raten von Progesteron sind bei Männern sowie bei schwangeren, nichtschwangeren und ovariektomierten Frauen etwa gleich groß. Wie auch bei den Ostrogenen ist die metabolische Clearance-Rate von Progesteron im Blut signifikant höher als im Plasma.

\section{Production rates and metabolic clearance rates of oestrogens and of progesterone in man}

Two procedures can be used for the determination of the production rates of oestrogens and of progesterone: The "urinary" production rate and the "blood" production rate. The "urinary" production rate ( $\mathrm{PR}_{\text {urine }}$ ) is calculated according to the formula

$$
\mathrm{PR}_{\mathrm{urine}}=\frac{\text { radioactive steroid injected }}{\text { specific activity of metabolite }} \times \frac{1}{\mathrm{t}} \text {, }
$$

the "blood" production rate according to

$$
\mathrm{PR}_{\text {blood }}=\mathrm{MCR} \times \mathrm{C},
$$

whereby MCR is the metabolic clearance rate and $C$ the concentration of endogenous steroid in the plasma. The PR $\mathrm{Prine}_{\mathrm{u}}$ of oestrogens is of rather limited value, since these steroids are subjected to enterohepatic circulation; furthermore oestrone and oestradiol-17 $\beta$ are interconverted in the organism, the rate of aromatization of androgens to oestrogens depends on numerous factors, and kidney and liver functions also play an important role. The $\mathrm{PR}_{\text {urine }}$ of oestrone and oestradiol-17 $\beta$ of nonpregnant women is lower during the follicular phase than the luteal phase; in males, the values are significantly lower than in females during the reproductive phase of their life. At the end of pregnancy, the combined $\mathrm{PR}_{\text {urine }}$ for oestadiol- $17 \beta$ and oestrone may be as high as $30 \mathrm{mg} / \mathrm{day}$. Only a few values have been published for $\mathrm{PR}_{\mathrm{blood}}$ owing to certain difficulties in the determination of endogenous oestrogen in plasma.

So far, metabolic clearance rates have been estimated by the technique of continuous infusion. The metabolic clearance rate of oestrone are similar for men and women, whereas the metabolic clearance rate of oestradiol-17 $\beta$ is significantly higher in males than in females. The value for the metabolic clearance rate depends on the hormonal status of the organism.

The calculation of the $P_{\text {urine }}$ of progesterone is based on the excretion of $5 \beta$-pregnane-3 $\alpha, 20 \alpha$-diol which is the main metabolite of progesterone. Since $5 \beta$-pregnane-3 $\alpha, 20 \alpha$-diol is not only formed from progesterone but also from other adrenal steroids, the determination of the $\mathrm{PR}_{\mathrm{urine}}$ of progesterone is only useful as a screening test during pregnancy, where the production of progesterone is high. The $\mathrm{PR}_{\text {blood }}$ of progesterone is estimated in the usual way by directly measuring the concentration of this steroid in plasma. The values for the $\mathrm{PR}_{\mathrm{blood}}(\mathrm{mg} / 24 \mathrm{~h})$ are 0.52 in men, 2.1 in women during follicular phase, 24.9 in women during luteal phase and 293 at the end of pregnancy.

The metabolic clearance rate values for progesterone are similar for men, pregnant, non-pregnant and oophorectomised women. As with oestrogens, the metabolic clearance rate for progesterone is significantly higher in blood than in plasma.

1) Vorgetragen auf dem Methoden-Kolloquium „Probleme der Bestimmung von Steroidproduktions- und Sekretionsraten“ am 1. März 1972 in Hannover anläßlich des 18. Symposiums der Deutschen Gesellschaft für Endokrinologie. 
Während die Begriffe „Steroidproduktionsrate“ und "Metabolische Clearancerate" eindeutig definiert sind, herrschen über die „Steroidsekretionsraten" hinsichtlich ihrer experimentellen Bestimmung, ihrer Interpretation und ihres Aussagewertes unterschiedliche Auffassungen (vgl. 1, 2). Bei genauer Auslegung ist festzustellen, daß die sogenannten Sekretionsraten nur dann verläßlich bestimmt werden können, wenn - bei gleichzeitiger Kenntnis der Durchblutungsrate - die Konzentrationsunterschiede für das jeweilige Steroid zwischen dem arteriellen und dem venösen Blut des endokrinen Organs gemessen werden. Alle Angaben von Sekretionsraten, die nicht auf diese Weise ermittelt werden, basieren auf mathematischen Überlegungen an idealisierten Denkmodellen (3). Der Vollständigkeit halber sei vermerkt, daß Pearlman (4) bereits 1957 überzeugende Definitionen der Sekretions- und Produktionsraten gegeben hat, die auch heute noch gültig sind. Aus den hier kurz dargelegten Gründen sowie im Hinblick auf die Unterschungen und Erkenntnisse der letzten Jahre werden im folgenden ausschließlich die Produktionsraten und metabolischen Clearanceraten der Östrogene und von Progesteron besprochen.

\section{„Urin“-Produktionsraten von Östrogenen}

Schon 1957 hat Brown (5) unter Verwendung von nichtmarkierten Östrogenen die Produktionsraten für Östron und Östradiol-17 $\beta$ während des Zyklus sowie für Östron, Östradiol-17 $\beta$ und Östriol während der Schwangerschaft ermittelt. Zu diesem Zwecke injizierte er nichtmarkiertes Östron, Östradiol-17 $\beta$ und/oder Östriol und bestimmte mit Hilfe der von ihm entwickelten Methode die Ausscheidung der drei genannten Steroide im Urin (6). Wie aus Abbildung 1 hervorgeht, beträgt die Produktionsrate von Östron und Östradiol$17 \beta$ zum Zeitpunkt der Ovulation etwa $350 \mu \mathrm{g} / 24 \mathrm{~h}$. Dieser Wert sowie die Angaben für die Follikel- und Lutealphasen liegen im gleichen Bereich wie die später

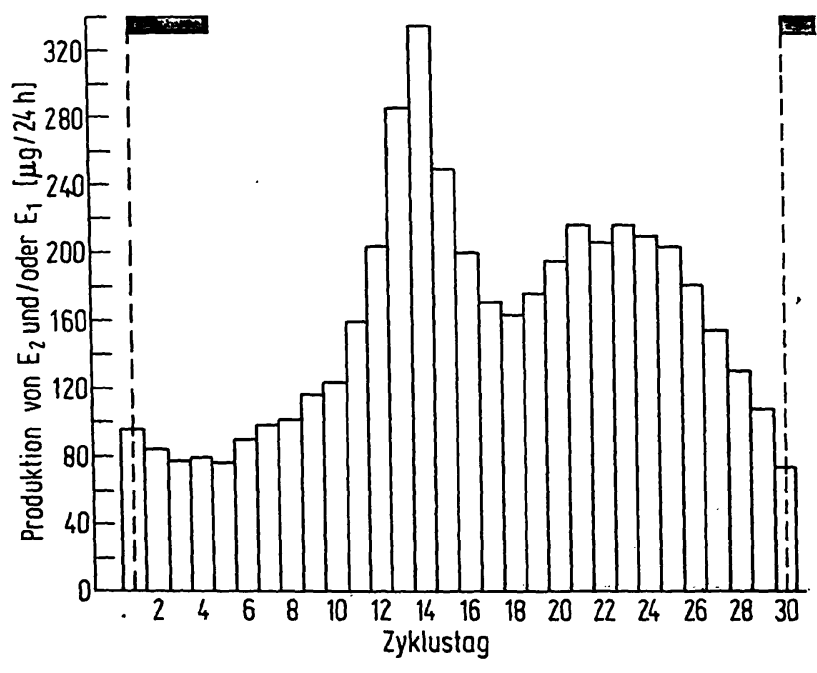

Abb. 1

Produktionsraten von Östrogenen während eines ovulatorischen menstruellen Zyklus. Die Werte sind als Ostradiol-17 $\beta$ und/oder Ostron ausgedrückt. Die Zahlen sind auf Grund der 24-h-Ausscheidung von Ostron, Ostradiol-17 $\beta$ und Ostriol im Urin bei 11 menstruellen
berechnet.
Menstruationsblutung. Nach BROWN (5)

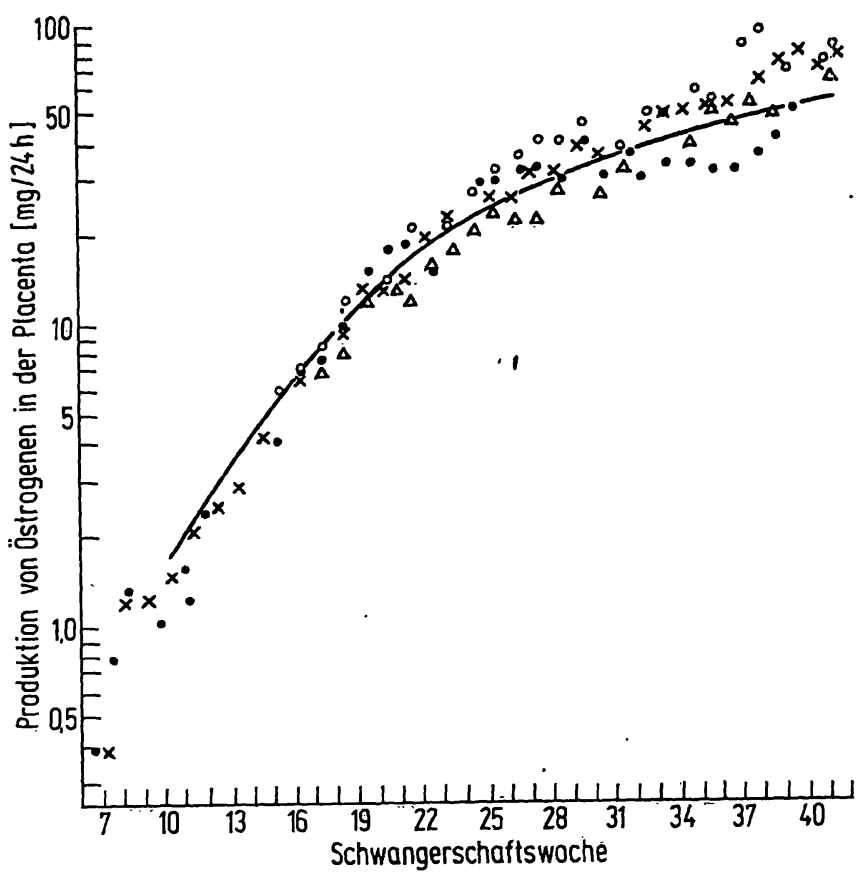

Abb. 2

Zeitlicher Verlauf der placentaren Sekretion der "Gesamtöstrogene" (Ostron, Ostradiol-17 $\beta$ und Ostriol) bei 4 normal veriaufenden Schwangerschaften. Die Kurvenpunkte wurden auf Grund von Ausscheidungswerten im Urin berechnet

Schwangere $1(\bullet), 2(x), 3(\Delta)$ und $4(0)$. Nach BRown (5)

mit radioaktiv markierten Steroiden ermittelten Produktionstaten. Für schwangere Frauen berechnete Brown (5) Produktionstaten von etwa $25 \mathrm{mg}$ Östron und Östradiol- $17 \beta$ sowie von etwa $65 \mathrm{mg}$ für Östriol. Der von Brown (5) angegebene zeitliche Verlauf der von der Placenta produzierten Gesamtöstrogene ist in Abbildung 2 wiedergegeben. Auch diese Werte stimmen im wesentlichen mit neueren Angaben, die unter Verwendung von deuterierten Östrogenen gewonnen wurden, überein (7).

Nach Einführung rádioaktiv markierter Steroide stehen heute zur Bestimmung der Produktionstaten von Östrogenen grundsätzlich zwei Verfahren zur Verfügung:

1. Die sogenannte Urin-Produktionsrate und

2. die sogenannte Blut-Produktionsrate.

Bei der Ermittlung der „Urin“-Produktionsrate wird ein radioaktiv markiertes Östrogen einmal intravenös injiziert; anschließend wird die kumulative Ausscheidung der radioaktiv markierten Metaboliten sowie der endogen produzierten Östrogene im Urin gemessen. Aus den so gewonnenen Werten wird die spezifische Aktivität des jeweiligen Metaboliten - z. B. von Östradiol-17 $\beta$ oder Östron - errechnet und die „Urin"Produktionsrate nach folgender Formel ermittelt:

$$
\mathrm{PR}_{\mathrm{Urin}}=\frac{\text { injiziertes radioaktiv markiertes Steroid }}{\text { spezif. Akt. des Metaboliten }} \times \frac{1}{\mathrm{t}} .
$$

Da zur quantitativen Bestimmung der endogenen Östrogene im Urin bereits seit längerer Zeit zuverlässige Methoden bekannt sind $(6,8,9,10)$ wurde die Produktion von Östrogenen bisher vorzugsweise als "Urin"Produktionsrate ermittelt. Die Aussagekraft der "Urin“Produktionsraten ist allerdings mit gewissen metho- 


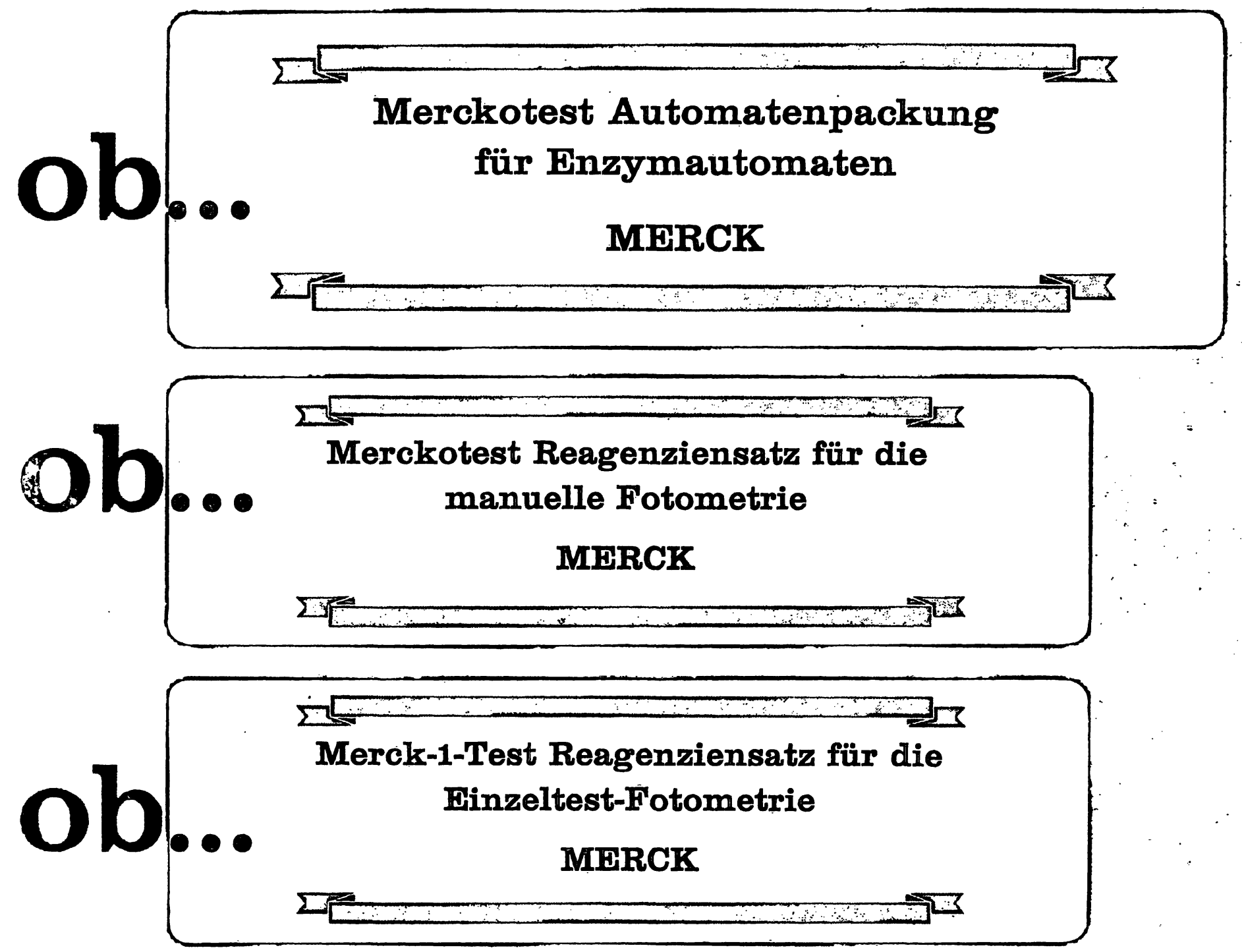

Merck hat alle Voraussetzungen geschaffen für die Vergleichbarkeit der Laborergebnisse in Klinik und Praxis durch standardisierte Enzymbestimmungen mit optimierten Tests:

Alkalische Phosphatase (AP)

Glutamat-dehydrogenase (GlDH)

Glutamat-Oxalacetat-Transaminase (GOT)

Glutamat-Pyruvat-Transaminase (GP'T)

$a$-Hydroxybutyrat-Dehydrogenase (HBDH)

Lactat-Dehydrogenase (LDH)

Leucin-Arylamidase (sog. LAP)

Spezialprospekte "Merckotest Automatenpackungen”, "Merckotest” und „Merck-1-Test” senden wir Ihnen gern auf Anforderung zu. 


\section{WW Dosierung

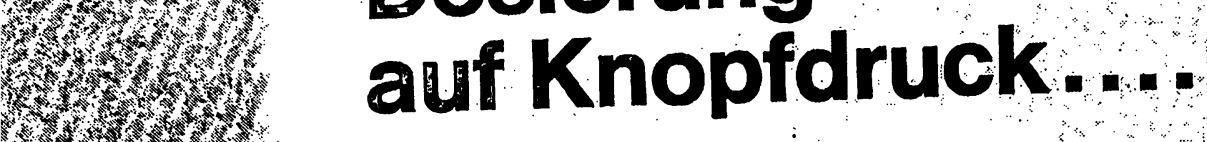

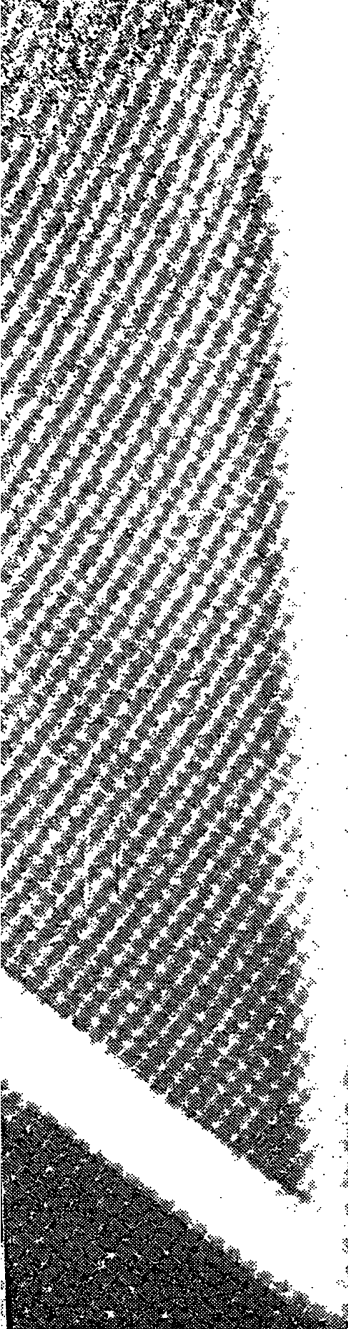

Hier ist der Weg, um von Zeit zu Zeit eine genaue Probenmenge durch Druck auf einen Knopf abzugeben Unsere PB 600 liefert bei jedem Knopfdruck 1/50 des Gesamtvolumens. Sie kann Spritzen von $10 \mathrm{ml}$ bis $0,25 \mathrm{ml}$ Volumen aufnehmen, Sie ist das ideale Gerät für Mikrotitrationen, Farbverdünnungen, Standardlösungen-und-Verdünnungen und Dünnschiehthtchromatographie. Eine Spezialausfưh hung für VDRL-Antigentests ist ebenfalls lieferbar:

Wann immer Sie also eine geringe Probenmenge brauchen und nur einen Knopf drúcken wollen oftragen Sie nach der HAMILTON PB 600 Ausführliche Informationen finden sie in unseren Katalog, den wir lhnen auf Anfrage gern zuschicken. Bitte schreiben Sie an Hamilton-Micromesure NV Postfach 205, Den Haag, Holland.
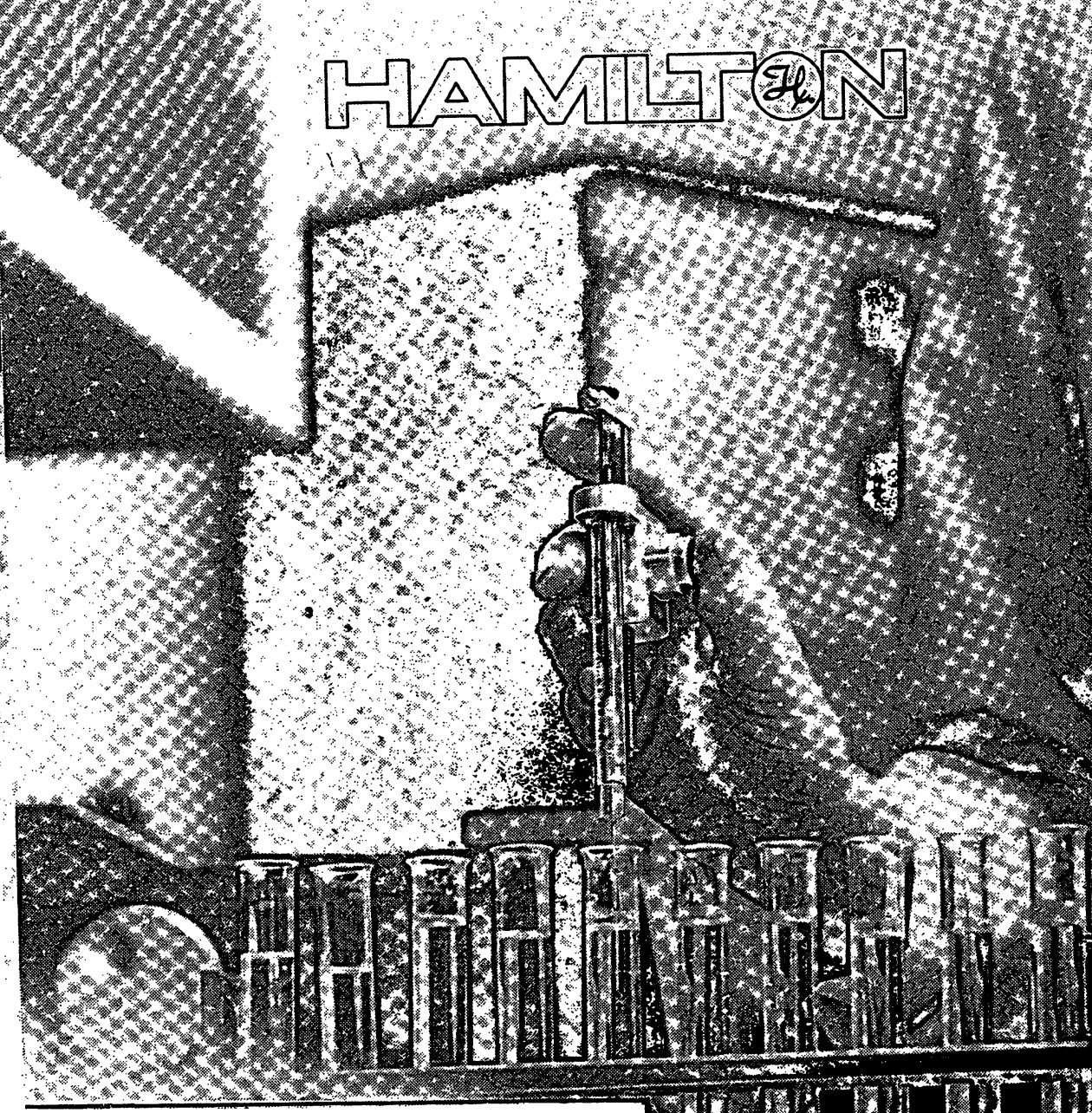

Autorisierter Händler für die BRD:

GÜNTHER SCHMIDT

Hamburg - Frankfurt

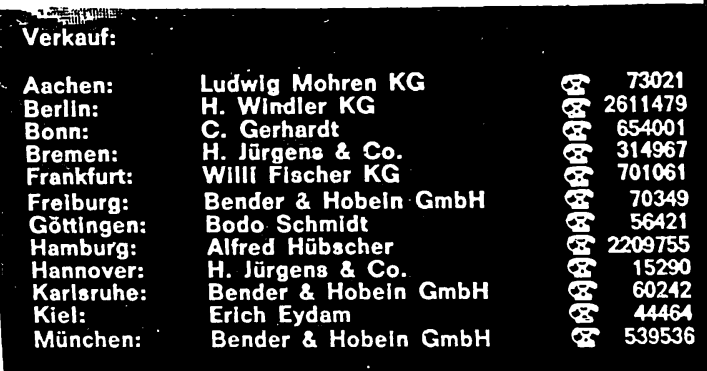

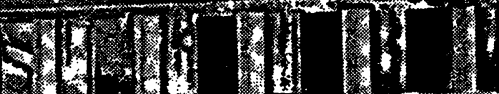
wro $+2$ 
dischen Unsicherheiten belastet. So haben bereits 1962 GuRPIDE und Mitarbeiter (11) Bedenken gegen die „Urin“-Produktionsraten angebracht - zu einem Zeitpunkt, als zwischen den Begriffen der Sekretionsrate und der Produktionsrate von zahlreichen Autoren noch nicht klar unterschieden wurde. Die Argumente von GURPIDE und Mitarbeitern (11) lassen sich hinsichtlich der Östrogene wie folgt zusammenfassen:

1. Die Östrogene unterliegen einem enterohepatischen Kreislauf, so daß ihr Stoffwechsel in nicht vorausschaubarer Weise durch die Darmflora beeinflußt werden kann (12).

2. Durch die Interkonversion von Östron und Östradiol$17 \beta$ wird unter Umständen eine zu hohe Produktionsrate vorgetäuscht.

3. Der Anteil von Östrogenen, der durch Aromatisierung von Androgenen entsteht, hat ebenfalls einen Einfluß auf die Höhe der errechneten Produktionsrate.

4. Schließlich sei darauf hingewiesen, daß der Stoffwechsel der Östrogene in der Schwangerschaft verschiedene Besonderheiten aufweist, so daß Berechnungen von Produktionsraten nicht möglich sind.

Daneben spielen bei der Bestimmung der „Urin“-Produktionsrate sowohl die Nierenfunktion als auch die Leberfunktion - insbesondere im Hinblick auf die Bildung von Konjugaten - eine wichtige Rolle; außerdem macht die unterschiedliche Clearance der einzelnen Östrogenkonjugate die Untersuchung der Urinproben über einen längeren Zeitraum notwendig. Bei der Interpretation der Urin-Produktionsraten wird im allgemeinen die stillschweigende Annahme gemacht, der Stoffwechsel von exogen zugeführten Östrogenen verhalte sich genau so wie derjenige der endogenen Östrogene; wie weit allerdings diese Annahme für die Östrogene zutrifft, ist bis heute nicht bewiesen.

In Tabelle 1 sind die „Urin"-Produktionsraten von Östrogenen bei nichtschwangeren Frauen zu verschiedenen Zeitpunkten des menstruellen Zyklus sowie bei
Männern zusammengestellt. Die „Urin“-Produktionsraten für Östradiol-17 $\beta$ sind nach den übereinstimmenden Angaben mehrerer Autoren während der Proliferationsphase (Follikelphase) niedriger als während der Sekretionsphase (Lutealphase). Inwieweit diese Unterschiede statistisch signifikant sind, läßt sich auf Grund der bisher vorliegenden Werte nicht entscheiden; einmal ist die Zahl der untersuchten Frauen zu gering, zum anderen wurden 4 verschiedene Bestimmungsmethoden benutzt. Es scheint jedoch kein Zweifel darüber zu bestehen, daß sowohl Östron und Östradiol$17 \beta$ als auch Östriol während des gesamten Zyklus produziert werden; dabei ist allerdings die Frage noch offen, in welchem Umfange Östriol direkt aus Östron bzw. Östradiol-17 $\beta$ oder aus anderen Vorstufen produziert wird.

Die „Urin"-Produktionsraten für Östradiol-17 $\beta$ und für Östron sind bei Männern deutlich kleiner als bei Frauen in der Reproduktionsphase. Die zum Teil erheblichen Unterschiede dürften kaum methodisch bedingt sein. Im übrigen scheinen nach den Ergebnissen von PREEDY und Mitarbeitern (15) zwischen Männern einerseits und Frauen in der ersten Zyklushälfte andererseits Differenzen im Produktionsmuster der Östrogene zu bestehen.

Wie bereits erwähnt, ergeben sich bei der Bestimmung der „Urin"-Produktionsraten von Östrogenen in der Schwangerschaft Interpretationsschwierigkeiten, die durch die foetoplacentare Einheit bedingt sind. GuRPIDE und Mitarbeiter (11) haben auf diesen Punkt besonders hingewiesen; auf Grund der 2-Kompartiment-Modellvorstellung folgern die Autoren, daß aus der Bestimmung der Östrogene und ihrer Metaboliten im Urin zwar auf die Sekretion der Östrogene in das mütterliche Kompartiment, nicht aber auf die endogene Produktionsrate geschlossen werden kann. Sofern diese Annahme zutrifft, repräsentieren die „Urin“-Produktionsraten für Östradiol- $17 \beta$ und Östron die von der Mutter und von der Placenta gebildeten Östrogene. Die

Tab. 1

„Urin"-Produktionsraten von Ostron $\left(E_{1}\right)$, Ostradiol-17 $\beta\left(E_{2}\right)$ und Ostriol $\left(E_{3}\right)$ bei nichtschwangeren Frauen und bei Männern. Bei Mittelwerten ist die Standardabweichung und die Zahl der Bestimmungen angegeben

\begin{tabular}{|c|c|c|c|c|c|c|c|}
\hline \multirow{2}{*}{$\begin{array}{c}\text { Frauen } \\
\text { (Zyklustag) } \\
\text { bzw. Männer }\end{array}$} & \multicolumn{7}{|c|}{ Produktionsraten in $\mu \mathrm{g} / 24 \mathrm{~h}$} \\
\hline & Ostrogen & $E_{1}$ & $\mathrm{E}_{2}$ & & $E_{3}$ & Autoren & Janr \\
\hline 6. & {$\left[{ }^{3} \mathrm{H}\right] \mathrm{E}_{2}$} & - & 104 & & - & [13] & 1963 \\
\hline 19. & & - & 151 & & - & & \\
\hline $6 .-10$. & {$\left[{ }^{36} \mathrm{C}\right] \mathrm{E}_{2}+\left[{ }^{3} \mathrm{H}\right] \mathrm{E}_{3}$} & - & 192 & & 12 & [14] & 1966 \\
\hline 19. -22 . & & - & 269 & & 23 & & \\
\hline 19. -22 . & & - & 151 & & 51 & & \\
\hline 5.- 8. & & - & 120 & & 24 & & \\
\hline $26 .-30$. & & - & 208 & & 44 & & \\
\hline 4. -8 & {$\left[{ }^{3} \mathrm{H}\right] \mathrm{E}_{1}+\left[{ }^{14} \mathrm{C}\right] \mathrm{E}_{2}$} & 104 & 130 & & - & [14] & 1966 \\
\hline $5 .-9$. & & 208 & 171 & & - & & \\
\hline $8 .-12$. & & 392 & 400 & & - & & \\
\hline $20 .-23$. & & 242 & 246 & & - & & \\
\hline 8. -12 . & {$\left[{ }^{3} \mathrm{H}\right] \mathrm{E}_{2}$} & - & $56 \pm 28(8)$ & & - & {$[15]$} & 1967 \\
\hline Männer & {$\left[{ }^{2} \mathrm{H}\right] \mathrm{E}_{2}$} & $\perp$ & $29 \pm 14(9)$ & & - & {$[17]$} & 1967 \\
\hline Männer & {$\left[{ }^{2} \mathrm{H}\right] \mathrm{E}_{2}$} & $23 \pm 7(6)$ & $32 \pm 13(6)$ & & - & {$[18]$} & 1970 \\
\hline
\end{tabular}


Tab. 2

„Urin"-Produktionsraten von Ostron $\left(E_{1}\right)$ und Ostradiol-17 $\beta\left(E_{2}\right)$ zu verschiedenen Zeiten der Schwangerschaft

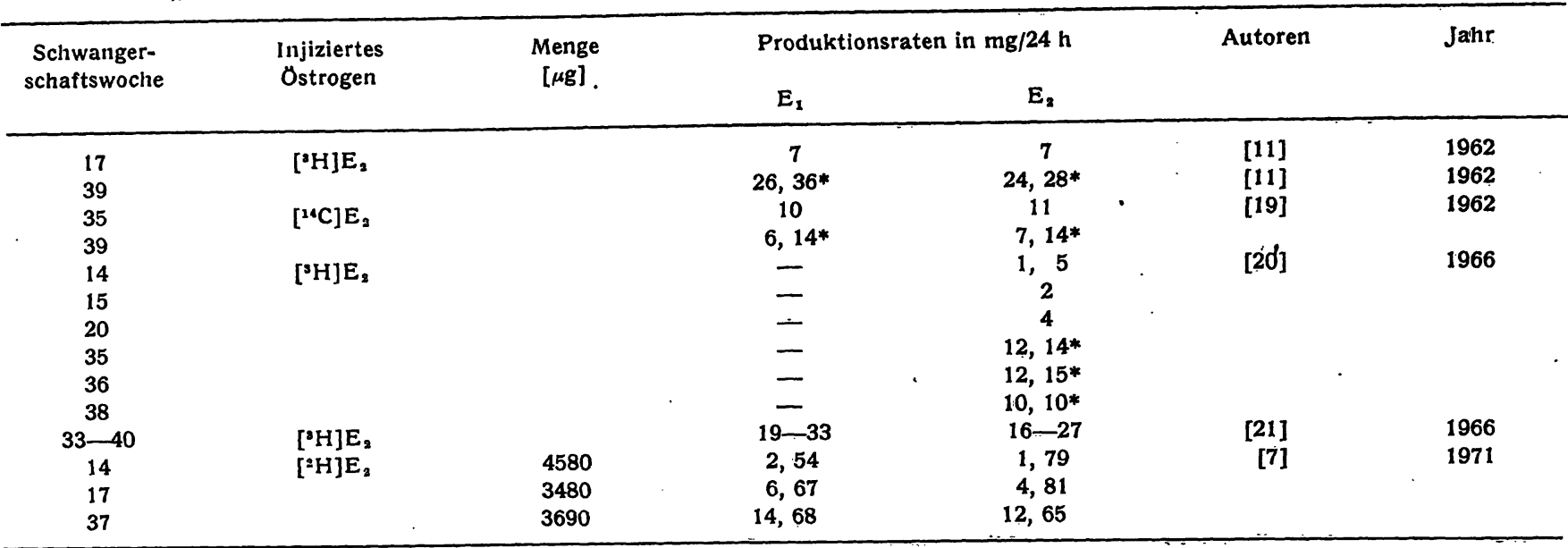

* Mittelwert von verschiedenen Schwangeren

Tab. 3

„Urin“-Produktionsraten von Ostron $\left(\mathrm{E}_{1}\right)$ und Ostradiol-17 $\beta\left[\mathrm{E}_{2}\right]$ unter verschiedenen pathologischen Bedingungen. Es sind die Mittelwerte, $\mathrm{z}$. T. die Standardabweichungen bzw. die Schwankungsbereiche sowie die Zahl der Bestimmungen angegeben

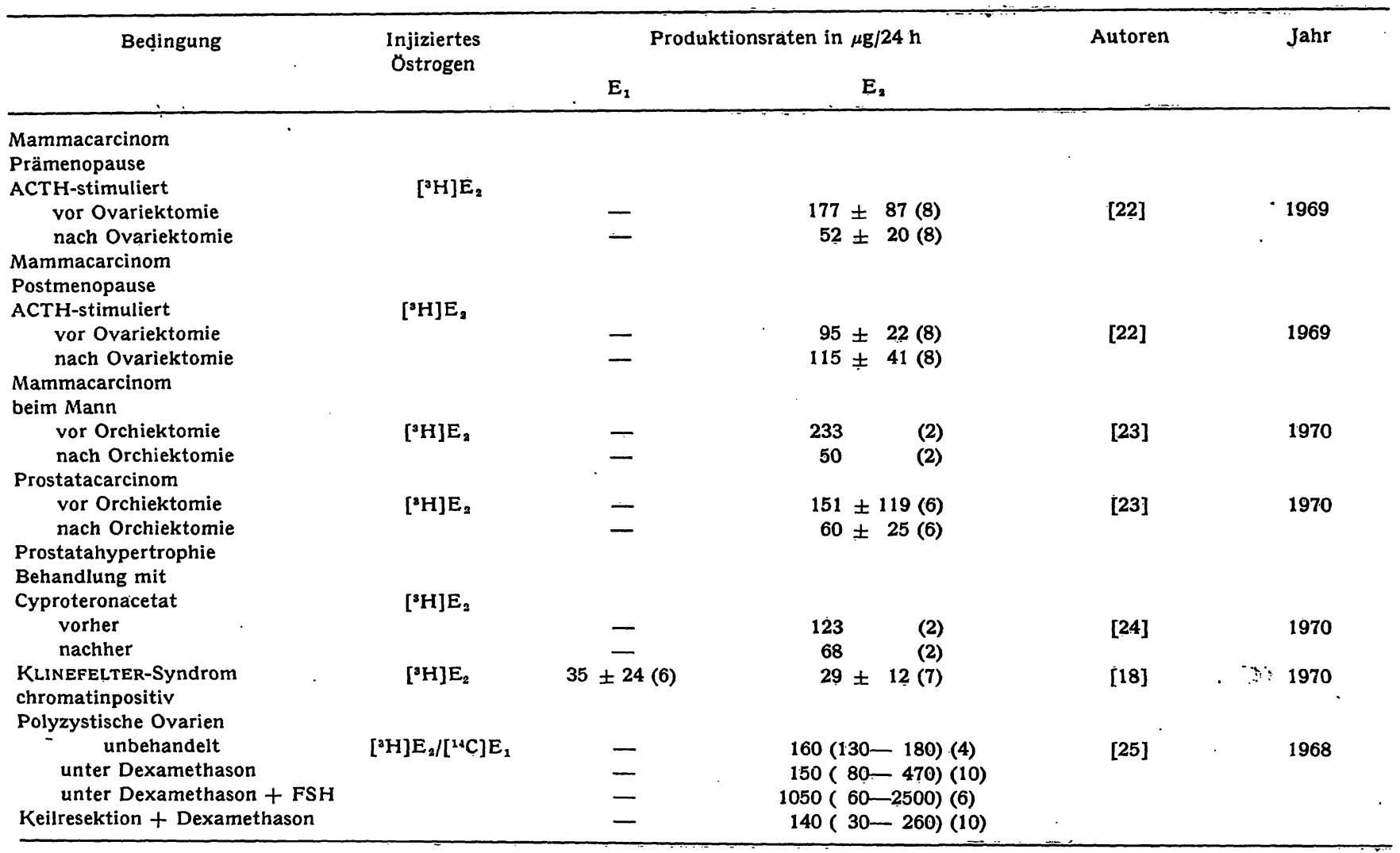

Messung von „Urin"-Produktionsraten während der Schwangerschaft ist bisher. unter Verwendung radioaktiv markierter Östrogene in $\mu \mathrm{g}$-Mengen erfolgt. Kürzlich konnten PINkUs und Mitarbeiter (7) zeigen, daß auch deuterierte Steroide, die in mg-Mengen zugeführt werden können, ebenfalls zur Messung von „Urin“Produktionsraten geeignet sind. In der Tat stimmen, wie aus Tabelle 2 hervorgeht, die mit den verschiedenen Methoden ermittelten Produktionsraten für Östron und Östradiol-17 $\beta$ während der Schwangerschaft weitgehend überein; am Ende der Schwangerschaft werden für die beiden Östrogene Werte bis zu etwa $30 \mathrm{mg} / 24 \mathrm{~h}$ beobachtet. Die Verwendung deuterierter Östrogene bei der Bestimmung von Produktionsraten erfordert zwar einen größeren apparativen Aufwand in Form eines Gaschromatograph/Massenspektrometers, hat aber den Vorteil der fehlenden Strahlenbelastung; diese kann bei Mehrfachuntersuchungen und bei Verabreichung größerer Steroidmengen nicht unerheblich sein.

Unter verschiedenen pathologischen Bedingungen sind ebenfalls die „Urin"-Produktionsraten vion Östrogenen gemessen worden. Einige wichtige Befunde sind in Tabelle 3 zusammengefaßt. Wie zu erwarten, sind die Produktionsraten bei Frauen in der Prämenopause und 


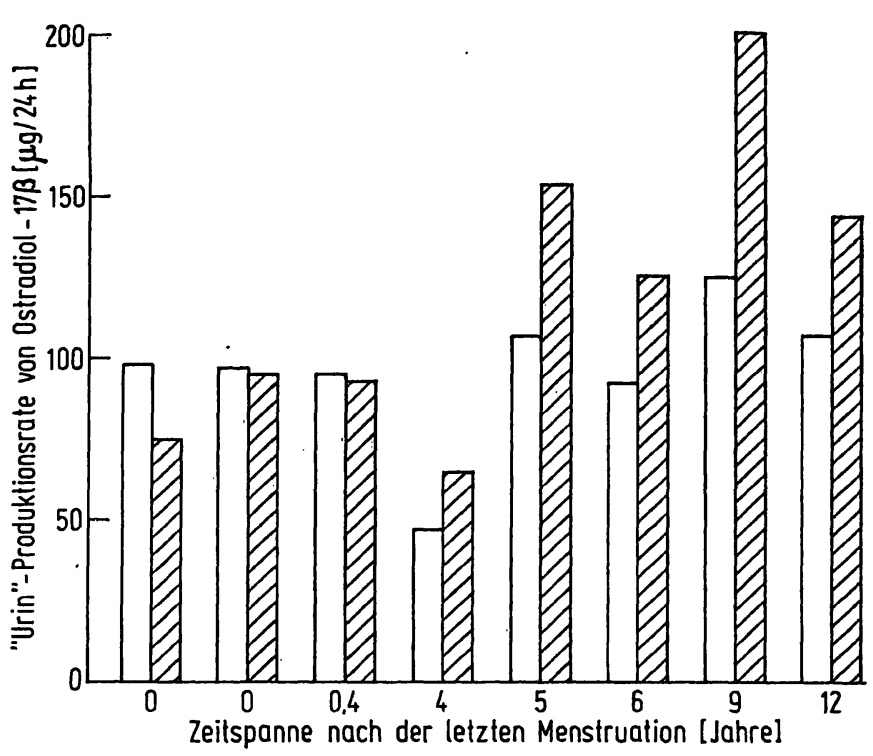

Abb. 3

"Urin"-Produktionsraten von Ostradiol-17 $\beta$ bei Frauen mit Mammacarcinom in der Postmenopause vor und nach Ovariektomie. Alle Werte wurden unter Stimulierung mit ACTH (40 I. U.) ermittelt. $\square$ vor Ovariektomie, $\square$ nach Ovariektomie Nach BARLOW und Mitarbeitern (22)

bei Männern vor Kastration deutlich größer als nach Kastration. Von besonderem Interesse ist die Beobachtung von Barlow und Mitarbeitern (22), daß die Produktionsraten von Östradiol-17 $\beta$ bei Frauen in der Postmenopause nach Ovariektomie eine Tendenz zum Anstieg erkennen lassen (Abb. 3). Dieser Anstieg wird mit zunehmendem Alter deutlicher und ist möglicherweise Ausdruck der ebenfalls zunehmenden Fähigkeit der Nebennierentinde, zur Produktion der Östrogene beizutragen - ein für die Therapie des Mammacarcinoms durchaus beachtenswerter Befund.

Bemerkenswert ist auch die Feststellung, daß die „Urin"-Produktionsrate von Östradiol-17 $\beta$ beim Manne durch Cyproteronacetat um etwa die Hälfte erniedrigt wird (24); diese Abnahme könnte sowohl durch eine verminderte Bildung von Östrogenvorstufen in den Testes als auch durch eine Hemmung der Aromatisierungsreaktion bedingt sein. Schließlich sei erwähnt, daß bei Frauen mit polycystischen Ovarien die Produktionsrate von Östradiol-17 $\beta$ durch Verabreichung von FSH auf das 7-8fache gesteigert wird.

\section{Metabolische Clearanceraten von Östrogenen}

Erst durch die Einführung radioaktiv markierter Steroide ist es möglich geworden, verläßliche Werte bei der Bestimmung der metabolischen Clearanceraten zu erhalten. Die metabolische Clearancerate (MCR) wird in ihrer allgemeinen Form folgendermaßen definiert:

$$
\mathrm{MCR}=\frac{\mathrm{R}}{\mathrm{x}^{\prime} \cdot \mathrm{dt}}
$$

Hierbei bedeuten:

$\mathbf{R}=$ eingesetzte Radioaktivität

$\mathbf{x}^{\prime}=$ Radioaktivität im Plasma

$\mathrm{t}=$ Zeit

Ohne auf weitere Einzelheiten einzugehen (vgl. (26)), seien die beiden gängigen Verfahren zur experimentellen Bestimmung der metabolischen Clearance-Rate angegeben. Im ersten Falle erfolgt eine einmalige intravenöse Injektion des radioaktiv markierten Steroids. Die Konzentration des injizierten Steroids nimmt exponentiell ab. Im zweiten Falle wird das radioaktiv markierte Steroid nach Verabreichung einer Initialdosis (priming dose) kontinuierlich infundiert, bis ein Gleichgewicht (steady state) erreicht ist. Die bisherigen Untersuchungen zur Bestimmung der metabolischen Clearance-Rate von Östrogenen sind unter Verwendung der Infusionstechnik durchgeführt worden. Die wesentlichen Befunde sind in Tabelle 4 wiedergegeben. LONGCOPE, LAYNE und TAIr (27) haben nach Verabreichung einer Initialdosis für etwa $120 \mathrm{~min}$ tritiiertes Östron oder Östradiol-17 $\beta$ infundiert und dabei festgestellt, daß die metabolische Clearance-Rate für Östron bei Männern und Frauen etwa gleich groß ist, während die metabolische ClearanceRate für Östradiol-17 $\beta$ bei Männern signifikant höher ist als bei Frauen.

Bei der Interpretation dieses Unterschiedes ist zu beachten, daß die metabolische Clearance-Rate eines Steroids um so höher liegt, je geringer seine Bindung an spezifische Plasmaproteine ist. Weder für Östron noch für Östradiol-17 $\beta$ wurde ein Unterschied in der metabolischen Clearance-Rate während der Follikelphase und der Lutealphase gefunden. Die Tatsache, daß die metabolischen Clearance-Raten im Blut höher liegen als im Plasma, ist durch die Bindung der Steroide an die

Tab. 4

Metabolișchẹ Clearance-Raten von Ostron und Ostradiol-17 $\beta$ bei gesunden Frauen und Männern

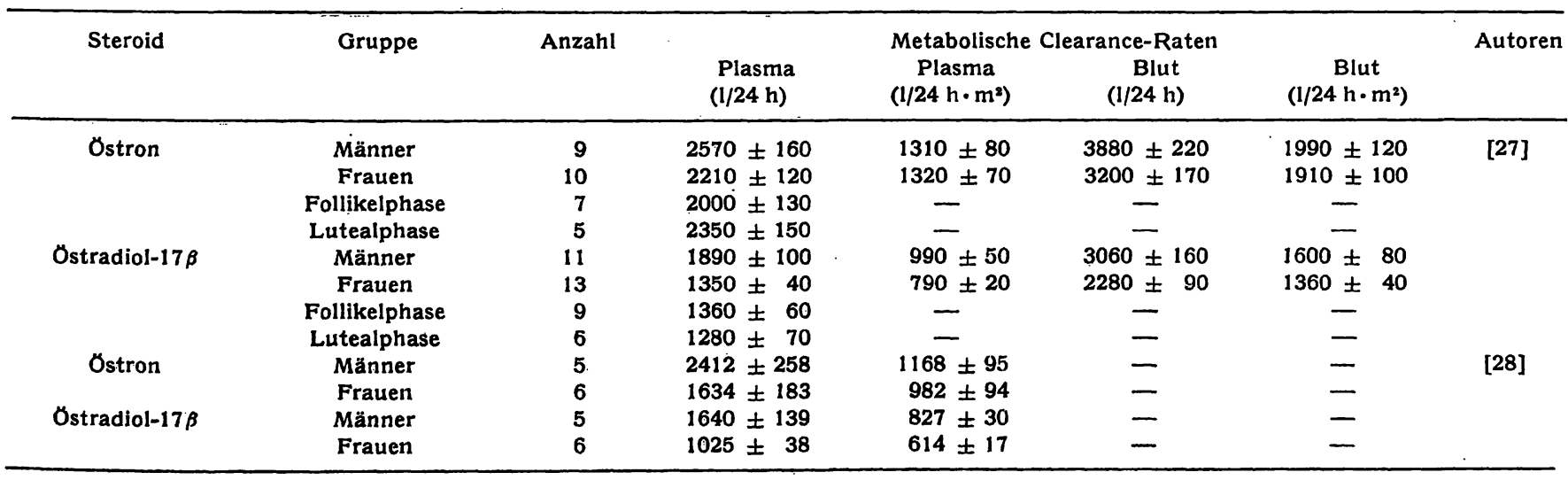




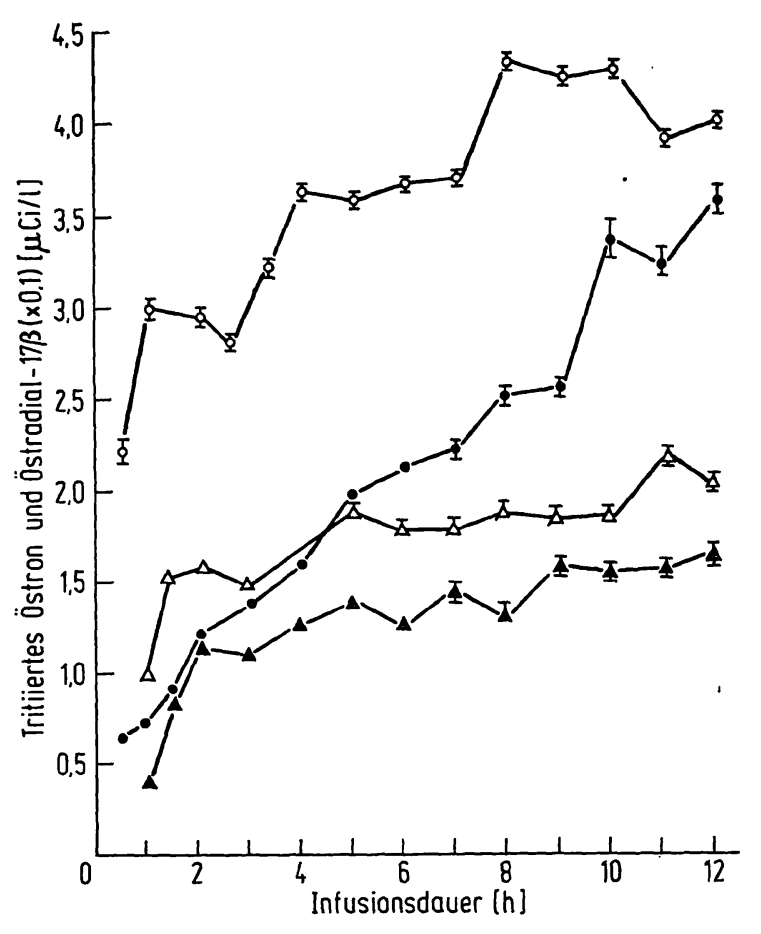

Abb. 4

Plasmakonzentration von tritiiertem Östron $(O, A)$ und Östradiol-17 $\beta$ $(\Theta, A)$ während einer $12 \mathrm{stdg}$. Infusion von tritiiertem Ostron Patientin mit Mammacarcinom (o, •), Intusionsrate $22,9 \mu \mathrm{Ci} / \mathrm{h}$ Patient mit Choriocarcinom $(\Delta, \Delta)$, Infusionsrate $16,1 \mu \mathrm{Ci} / \mathrm{h}$ Nach HEMBREE, BARDIN und LIPSETT (28)

Erythrocyten zu erklären. Auch LIPSETr und Mitarbeiter (28) fanden für Östradiol-17 $\beta$ bei Männern höhere Werte für die metabolischen Clearance-Raten als bei Frauen. Obgleich die Angaben dieser Autoren im wesentlichen mit denjenigen von LONGCOPE, LAYNE und TAIT (27) übereinstimmen, bestehen zwischen beiden Arbeitsgruppen gewisse Meinungsverschiedenheiten über die Infusionsdauer, die notwendig ist, um ein "steady state " für die Steroide zu erreichen. Nach den Untersuchungen von LIPSETT und Mitarbeitern (28) soll ein "steady state“ unter bestimmten Bedingungen entweder überhaupt nicht oder erst nach mehreren Stunden erreicht werden (Abb. 4). Die Validität dieser nicht unwichtigen Aussage wird von Longcope und TATT (29) aus drei Gründen angezweifelt:

1. Die Versuche sind nur an zwei Personen durchgeführt worden.

2. Bei diesen Personen handelte es sich um Patienten mit einem Mammacarcinom, bei denen mit einem veränderten Stoffwechsel der Östrogene gerechnet werden muß. 3. Die beobachteten Schwankungen können sowohl positiv als auch negativ verlaufen und dürften Ausdruck einer individuell variierenden Durchblutung der Leber sein.

In der Tat konnten Longcope und TAIT (29) über einen Zeitraum von $750 \mathrm{~min}$ bei einer größeren $\mathrm{Zahl}$ von gesunden Versuchspersonen folgende prozentuale Schwankungen - bezogen auf den 150 min-Wert finden: Für Östron von $-7,4$ bis $+9,0$ bei einem Variations-Koeffizienten von $11,5 \%$ und für Östradiol$17 \beta$ von $-10,6$ bis $+4,3$ bei einem Variations-Koeffizienten. von $10,0 \%$. Aus diesen Versuchen kann ge-
Tab. 5

Metabolische Clearance-Raten von Ostron und Ostradiol-17 $\beta$ bei gesunden Frauen, bei Frauen in der frühen $(<1 \mathrm{Jahr})$ und in der späten Menopause ( $>3$ Jahre) sowie bei hohem Gehalt an Corticosteroiden. Einzelheiten vgl. [28]

\begin{tabular}{cccc}
\hline Frauen & Anzahl & $\begin{array}{c}\text { Metabolische Clearance-Raten } \\
\left(1 / 24 \mathrm{~h} \cdot \mathrm{m}^{2}\right) \\
\text { Ostradiol-17 } \beta\end{array}$ \\
\hline Reproduktionsphase & 6 & $982 \pm 94$ & $614 \pm 17$ \\
Frühe Menopause & 3 & $987 \pm 60$ & $732 \pm 18$ \\
Späte Menopause & 3 & $119 \pm 73$ & $1054 \pm 48$ \\
Corticosteroidüberschuß & 3 & $119 \pm 19$ & \\
\hline
\end{tabular}

schlossen werden, daß - von speziellen Ausnahmen abgesehen - eine 2stündige Infusionsdauer ausreicht, um verläßliche Werte für die metabolischen ClearanceRaten von Östron und Östradiol-17 $\beta \mathrm{zu}$ gewinnen.

Ein veränderter hormoneller Status scheint die metabolischen Clearance-Raten von Östron und Östradiol-17 $\beta$ $\mathrm{zu}$ beeinflussen (28). Wie Tabelle 5 zeigt, liegen die metabolischen Clearance-Raten für Östradiol-17 $\beta$ in der frühen Menopause höher als in der späten Menopause. Bei einem Glucocorticoid-Überschuß (Behandlung von Patientinnen mit Mammacarcinom mit Glucocorticosteroiden bzw. Patientin mit Cushrng-Syndrom) ist die metabolische Clearance-Rate erhöht; die gleiche Beobachtung wurde bei einer Patientin nach Behandlung mit Fluoxymesteron gemacht. Möglicherweise interferieren die Corticosteroide mit der Bindung zwischen Östradiol-17 $\beta$ und dem Plasmaeiweiß.

\section{„Blut"“-Produktionsraten für Östrogene}

Aus der metabolischen Clearance-Rate (MCR) und der endogenen Konzentration im Plasma (C) kann die „Blut“Produktionsrate von Östrogenen nach folgender Formel ermittelt werden

$$
\mathrm{PR}_{\mathrm{Blut}}=\mathrm{MCR} \times \mathrm{C}
$$

Bisher liegen kaum Werte für die „Blut"-Produktionsraten von Östrogenen vor. Dies beruht auf der derzeitigen - wenn auch oft unausgesprochenen Schwierigkeit, endogenes Östradiol-17 $\beta$ ünd insbesondere Östron im Plasma quantitativ zu bestimmen. SCIARRA und Mitarbeiter (30) ermittelten bei 3 Männern „Blut"-Produktionsraten für Östradiol-17 $\beta$ von 57,8, 66,8 und $61,1 \mu \mathrm{g} / 24 \mathrm{~h}$. Diese Werte liegen etwa doppelt so hoch wie die von anderen Autoren $(17,18)$ gemessenen „Urin“-Produktionsraten für Östradiol-17 $\beta$.

\section{„Urin"-Produktionsraten von Progesteron}

Die direkte Bestimmung der "Urin“-Produktionsraten von Progesteron stößt wegen der extrem geringen Ausscheidung dieses Steroids im Urin auf erhebliche Schwierigkeiten; dies gilt besonders für nichtschwangere Frauen und Männer. Da als Hauptmetabolit von Progesteron im Urin 5 $\beta$-Pregnan-3 $\alpha, 20 \alpha$-diol ausgeschieden wird, basieren alle Berechnungen auf diesem Metaboliten. So errechnete Peariman (31) unter Verwendung von $\left[16-{ }^{3} \mathrm{H}\right]$ Progesteron für die Spätschwangerschaft eine 
Produktionsrate von $250 \mathrm{mg} / 24 \mathrm{~h}$. Romanoff und Mitarbeiter (32) errechneten - ebenfalls auf der Basis der Pregnandiol-Ausscheidung - für Männer „Urin“Produktionsraten von 3,2 bis $7,4 \mathrm{mg}$ bei einem Mittelwert von $5,1 \mathrm{mg} / 24 \mathrm{~h}$. Ohne auf weitere Einzelheiten einzugehen sei darauf hingewiesen, da $\beta 5 \beta$-Pregnan- $3 \alpha$, $20 \alpha$-diol nicht nur ein Metabolit von Progesteron ist, sondern erhebliche Mengen dieses Steroids auch aus anderen Vorstufen entstehen, die in der Nebenniere gebildet werden und bei denen es sich z. B. um $3 \beta$ Hydroxy-5-pregnen-20-on und dessen Sulfat handelt. Die Bestimmung der „Urin"-Produktionsraten von Progesteron ist deshalb nur dann sinnvoll, wenn die endogene Produktion von Progesteron so gro $\beta$ ist, $\mathrm{da} \beta$ die Menge an Pregnandiol, die aus anderen Vorläufern gebildet wird, vernachlässigt werden kann. Diese Bedingung trifft im wesentlichen für die Schwangerschaft, daneben aber auch bei Frauen in der Lutealphase des menstruellen Zyklus zu. Demnach hat die Bestimmung der „Urin"-Produktionsrate von Progesteron nur während der Schwangerschaft als „Screening“-Test einen Aussagewert (vgl. 33).

\section{Metabolische Clearance-Raten und „Blut"-Produk- tionsraten von Progesteron}

Die metabolischen Clearance-Raten von Progesteron sind bei Männern sowie bei schwangeren, nichtschwangeren und ovariektomierten Frauen etwa gleich groß (Tab. 6). Dieser Befund spricht in gewisser Weise gegen die Anwesenheit eines Plasmaproteins, das spezifisch Progesteron bindet. Progesteron wird bei $37^{\circ}$ an gereinigtes Transcortin mit einer Assoziationskonstanten von $3 \times 10^{7} 1 / \mathrm{Mol}$ und an das saure $\alpha_{1}$-Glykoprotein mit einer Assoziationskonstanten von 3,7 $\times 10^{5} 1 / \mathrm{Mol}$ gebunden (34). $\mathrm{Da}$ Transcortin sowohl Cortisol als auch Progesteron bindet, dürfte das Ausmaß der enterohepatischen Extraktion (splanchnic extraction) von Progesteron im wesentlichen durch die Menge an freiem und an Albumin gebundenem Progesteron bedingt sein (33). Obgleich in der Schwangerschaft die Menge an Transcortin vermehrt ist und infolgedessen auch die Bindung von Progesteron erhöht sein sollte, erfolgt dennoch keine Änderung der metabolischen Clearance-Rate von Progesteron. Dies spricht für eine erhöhte extra-enterohepatische Extraktion von Progesteron, eine Vermutung, die noch der experimentellen Nachprüfung bedarf. Wie auch bei den Östrogenen ist die metabolische Clearance-Rate $(1 / 24 \mathrm{~h})$ für Progesteron im Blut mit $3691 \pm 119$ signifikant höher als im Plasma mit $2178 \pm 194$ (33).
Tab. 6

Metabolische Clearance-Raten (MCR) und ,Blut"-Produktionsraten (PRBlut) für Progesteron bei Männern und Frauen [33]. Die Zahlen in der Tabelle geben die Mittelwerte, die Standardabweichungen und die Zahl der Versuche an

\begin{tabular}{|c|c|c|c|c|}
\hline Gruppe & $\operatorname{MCR}(1 / 24 h)$ & $\begin{array}{c}\text { Konzentr } \\
\text { von Proges } \\
\text { im Plas } \\
\text { (ng/100 }\end{array}$ & $\begin{array}{l}\text { ation } \\
\text { teron } \\
\text { ma } \\
\mathrm{ml} \text { ) }\end{array}$ & $\begin{array}{l}\text { PRBlut } \\
(\mathrm{mg} / 24 \mathrm{~h})\end{array}$ \\
\hline $\begin{array}{l}\text { Männer } \\
\text { Frauen }\end{array}$ & $2219 \pm 175(16)$ & $28 \pm 4$ & (13) & $0,52 \pm 0,05$ \\
\hline $\begin{array}{l}\text { Ovariektomie } \\
\text { Zyklus }\end{array}$ & $2228 \pm 137(14)$ & $38 \pm 4$ & (6) & $0,80 \pm 0,07$ \\
\hline Follikelphase & 2200 & 95 & (26) & 2,1 \\
\hline Lutealphase & 2200 & 1130 & (29) & 24,9 \\
\hline Schwangerschaft & $2352 \pm 151(24)$ & $12460 \pm 130$ & $(12)$ & $293,1 \pm 29,6$ \\
\hline
\end{tabular}

Wie bereits angedeutet, ist die Bestimmung und Interpretation der „Urin“-Produktionsraten von Progesteron mit Schwierigkeiten behaftet. Aus diesem Grund wird den Werten der „Blut"-Produktionsraten eine größere Richtigkeit und damit auch eine größere Aussagekraft zugemessen. Die Bestimmung der „Blut“-Produktionsraten von Progesteron stützt sich ausschließlich auf die direkte Bestimmung dieses Steroids im Plasma, so daß eine indirekte Berechnung über Pregnandiol - wie im Falle der „Urin"-Produktionsraten - nicht notwendig ist. Diese Tatsache erklärt die Differenzen zwischen den Werten der „Urin"- und der „Blut"-Produktionsraten für Progesteron insbesondere bei Männern sowie bei Frauen in der Follikelphase oder nach Ovariektomie und in der Menopause. Wie aus Tabelle 6 hervorgeht, betragen die "Blut"-Produktionsraten für Progesteron bei Männern $0,52 \mathrm{mg} / 24 \mathrm{~h}$, bei Frauen in der Follikelphase $2,1 \mathrm{mg} / 24 \mathrm{~h}$, in der Lutealphase $24,9 \mathrm{mg} / 24 \mathrm{~h}$ und am Ende der Schwangerschaft $293 \mathrm{mg} / 24 \mathrm{~h}$. Der letztgenannte Wert zeigt, daß bei einer größeren Produktion von Progesteron eine weitgehende Übereinstimmung zwischen den „Urin"- und „Blut"-Produktionsraten besteht.

Die hier beschriebenen Befunde über die Produktionsraten und metabolischen Clearance-Raten lassen deutlich werden, daß unsere heutigen Kenntnisse zwar noch lückenhaft sind, andererseits aber auf Grund der inzwischen entwickelten Techniken die berechtigte Hoffnung besteht, weitere Einblicke in dieses wichtige und interessante Gebiet der Steroiddynamik zu gewinnen. Insbesondere wird sich die Forschung der Aufklärung derjenigen exogenen und endogenen Faktoren zuwenden müssen, die einen Einfluß auf die Größe der metabolischen Clearance- und Produktionsraten ausüben und somit für die Erhaltung oder Störung des Hormongleichgewichtes beim Menschen verantwortlich sind.

\section{Literatur}

1. Methodenkolloquium „Möglichkeiten und Grenzen der Analytik mit isotopenmarkierten Steroiden" am 3. März 1971 in Hạnburg anläßlich des 17. Symposiums der Deutschen Gescllschaft für Endokrinologie. - 2. BreuER, H. und L. Nocke, Mitteilungen d. Deutschen Gesellschaft für Klinische Chemie, Heft 3, 1971. 3. Laumas, K. R., J. F. Tart und S. A. S. Tait, Acta endocr.
(Kbh.) 36, 265 (1961). - 4. Pearldman, W. H., Ciba Foundation Coll. Endocr. 11, 233 (1957). - 5. Brown, J. B., J. Endocr. 16, 202 (1957). - 6. Brown, J. B., Biochem. J. 60, 185 (1955). 7. Pinkus, J.I., D. Cinarless und S. C. Chattoraj, J. biol. Chemistry 246, 633 (1971). - 8. Brown, J. B., R. D. Bulbrook und F. C. Greenwood, J. Endocr. 16, 49 (1957). - 9. Preedy, 
J. R. K. und E. H. ArtKen, J. biol. Chemistry 236, 1300 (1961). 10. Barlow, J. J., Analyt. Biochem. 6, 435 (1963). - 11. Gurprde, E., M. Angers, R. L. VandeWrele und S. Lieberman, J. clin. Endocr. Springfield 22, 935 (1962). - 12. SANDBERG, A. A. und W. R. SLAunwhite, Jr., J. clin. Invest. 36, 1266 (1957). - 13. Morse, W. I., A. F. Clark und S. C. Maclead, J. Endocr. 26, 25 (1963). - 14. Barlow, J. J. und C. M. LogaN, Steroids 7, 309 (1966). - 15. Eren, S., G. H. Reynolds, M. E. Turner, Jr., F. H. SchmidT, J. H. MACKAY, C. M. Howard und J. R. K. Preedy, J. clin. Endocr. Springfield 27, 1451 (1967). - 16. Crowell, G. C., M. E. Turner, Jr., F. H. Schmidt, C. M. Howard und J. R. K. Preedy, J. clin. Endocr. Springfield 27 807 (1967). - 17. EREN, S., G. H. Rexnolds, M. E. Turner, Jr., F. H. Schmidt, J. H. MacKay, C. M. Howard und J. R. K. Preedy, J. clin. Endocr. Springfield 27, 819 (1967). - 18. GabriIOVE, J. L., G. L. Nrcolis und R. U. HAUSKNECHT, Acta endocr. (Kbh.) 63, 499 (1970). - 19. Fishman, J., J. B. Brown, L. Hellman, B. Zumoff und T. F. Gallagher, J. biol. Chemistty 237. 1489 (1962). - 20. SIrTeri, P. K. und P. C. MACDONALD, J. clin. Endocr., Springfield 26, 751 (1966). - 21. HobkiRk, R. und M.
NILSEN, J. clin. Endocr., Sptingfield 26, 625 (1966). - 22. BARLow, J. J., K. Emerson, Jt., und B. N. SAXiena, N. Engl. J. Med. 280, 633 (1969). - 23. HeLLMAN, L. und J. Fishman, J. Endocr. 46, 113 (1970).-24. Fishiman, J. und J. Geller, Steroids 16, 351 (1970). -25. JefFconte, S. L., R. V. Brooks, D. R. London, P. M. SMith, G. S. SpAThis und F. T. G. PRUNTY, J. Endoct. 42, 213 (1968). 26. TArr, J. F., J. clin. Endoct., Springfield 23, 1285 (1963). - 27. Longcope, C., D. S. LAYNE und J. F. TAIT, J. clin. Invest. 47, 93 (1968). - 28. HeMrreE, W. C., C. W. Bardin und M. B. LipSETT, J. clin. Invest. 48, 1809 (1969). - 29. LongCOPE, C. und J. F. TaIT, J. clin. Endocr., Springfield 132, 481 (1971). - 30. Concolino, G., A. Marocchi, S. Sorcint und F. Sctarra, Folia Endocrinol. 23, 399 (1970). - 31. Pearlman, W. H., Biochem. J. 65, 7P (1957). - 32. Romanoff, L. P., M. P. GRACE, M. N. BAXTeR und G. Panxus, J. clin. Endocrinol., Springfield 26, 1023 (1966). 33. Lrtrie, B. und R. B. Billiar, Excerpta Medica Internat. Congress Series 184, 871 (1969). - 34. SEAL, U. S. und R. P. DoE, In: Steroid dynamics p. 63 (G. Pincus, T. Nakao und J. F. TAIT, eds.) Academic Press, New York und London (1966).

Prof. Dr. H. Breuer D-53 Bonn-Venusberg

Inst. f. Klin. Biochemie und Klin. Chemie 